\title{
Bayesian Symbol Detection in Wireless Relay Networks via Likelihood-Free Inference
}

\author{
Gareth W. Peters ${ }^{1,2}$, Ido Nevat ${ }^{3}$, Scott A. Sisson ${ }^{1}$, Yanan Fan ${ }^{1}$ and Jinhong Yuan ${ }^{3}$ \\ 1 School of Mathematics and Statistics, University of New South Wales, Sydney, 2052, Australia; \\ email: peterga@maths.unsw.edu.au \\ 2 CSIRO Sydney, Locked Bag 17, North Ryde, New South Wales, 1670, Australia \\ 3 School of Electrical Engineering and Telecommunications, University of New South Wales, Sydney, Australia.
}

\begin{abstract}
This paper presents a general stochastic model developed for a class of cooperative wireless relay networks, in which imperfect knowledge of the channel state information at the destination node is assumed. The framework incorporates multiple relay nodes operating under general known non-linear processing functions. When a non-linear relay function is considered, the likelihood function is generally intractable resulting in the maximum likelihood and the maximum a posteriori detectors not admitting closed form solutions. We illustrate our methodology to overcome this intractability under the example of a popular optimal non-linear relay function choice and demonstrate how our algorithms are capable of solving the previously intractable detection problem. Overcoming this intractability involves development of specialised Bayesian models. We develop three novel algorithms to perform detection for this Bayesian model, these include a Markov chain Monte Carlo Approximate Bayesian Computation (MCMC-ABC) approach; an Auxiliary Variable MCMC (MCMC-AV) approach; and a Suboptimal Exhaustive Search Zero Forcing (SES-ZF) approach. Finally, numerical examples comparing the symbol error rate (SER) performance versus signal to noise ratio (SNR) of the three detection algorithms are studied in simulated examples.
\end{abstract}

\section{BACKGROUND}

Cooperative communications systems, [1] and [2], have become a major focus for communications engineers. Particular attention has been paid to the wireless network setting, as it incorporates spatial resources to gain diversity, and enhance connection capability and throughput. More recently, the focus has shifted towards incorporation of relay nodes [3], which are known to improve energy efficiency, and reduce the interference level of wireless channels [4].

In simple terms, such a system broadcasts a signal from a transmitter at the source through a wireless channel. The signal is then received by each relay node and a relay strategy is applied before the signal is retransmitted to the destination. A number of relay strategies have been studied in the literature ([5]; [2]). We focus on the amplify-and-forward strategy of [6] in which the relay sends a transformed version (determined by the relay function) of its received signal to the destination. The relay function can be optimized for different design objectives ([7]; [8]; [9]). It is common in the relay network literature to consider non-linear relay function choices which satisfy some concept of optimality. For example, in the estimate and forward (EF) scheme, in the case of BPSK signaling, the optimal relay function is the hyperbolic tangent [10].
Other criteria for which the optimal relay function is nonlinear include: capacity maximisation [10], minimum error probability at the receiver [11], SNR maximisation [9], rate maximisation [12] and minimisation of the average error probability [13].

Currently, in all these cited works the authors have solved the problem of what the optimal choice of the non-linear relay function should be in order to satisfy the desired design constraint. However, we note that in all cases, whenever a non-linear relay function is considered, though it may satisfy the constraint of optimality, it will result in an intractable detection problem. This intractability has not been considered in the literature and therefore it has not been possible to tackle the resulting detection problem. We specify explicitly how this intractability arises and then develop and demonstrate extensively our solution to this general relay network detection problem. In this paper we will demonstrate that the choice of relay function directly affects the tractability of the system model. We will focus on a single hop relay design in which the number of relays and the type of relay function are allowed to be known, general non-linear functions. However, our methodology trivially extends to arbitrary relay topologies and multiple hop networks. Fig. 1 presents the system model considered. Our model incorporates stochastic parameters that are associated with each relay channel link. That is, we consider parameters associated with the model as random variables, which must be jointly estimated with the unknown random vector of transmitted symbols.

Since this framework incorporates general relay functions, the resulting likelihood for the model typically cannot be evaluated pointwise, and in some cases cannot even be written down in a closed form. Bayesian "likelihood-free" inference methodologies overcome the problems associated with the intractable likelihood by replacing explicit evaluation of the likelihood with simulation from the model. These methods are also collectively known as Approximate Bayesian Computation (ABC), see [14], [15], [16], [17], [18], [19], and references therein for a detailed overview of the methodological and theoretical aspects.

Applications of ABC methods are becoming widespread, and include: telecommunications [18]; extreme value theory [20]; protein networks, ([14], [21]); SIR models [22]; species migration [23]; pathogen transmission [24], non-life insurance claims reserving [16]; and operational risk [25]. 


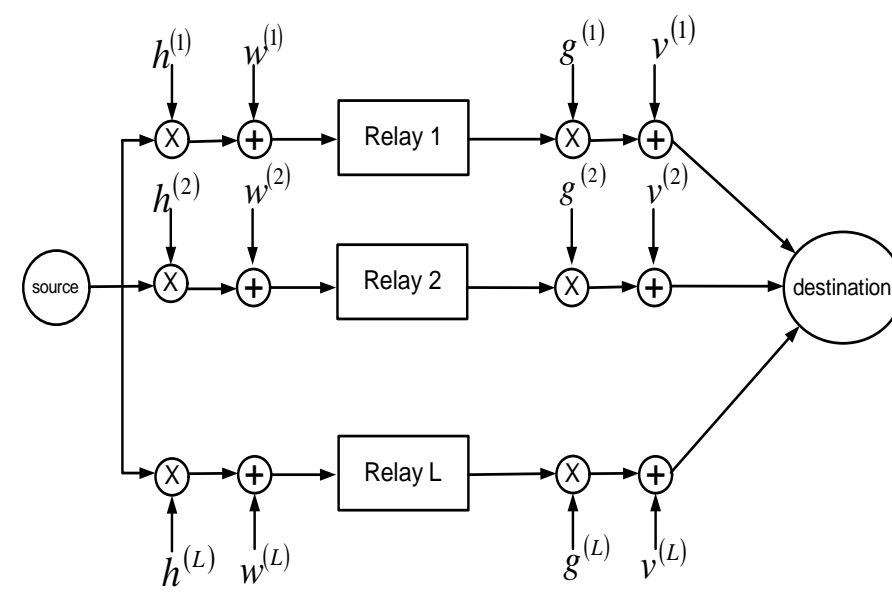

Fig. 1. The system model studied in this paper involves transmission from one source, through each of the $L$ relay channels, $h^{(l)}$, to the relay. Additive complex Gaussian noise, $\mathbf{W}$ was included at the receiver of the relay then the signal is processed and retransmitted by the relay to the destination. In this process the signal is transmitted again through $L$ channels denoted by $g^{(l)}$ and additive complex Gaussian noise, $\mathbf{V}$ was included at the destination.

\section{A. Main contributions}

In this paper we develop a novel sampling methodology based on the Markov Chain Monte Carlo Approximate Bayesian Computation (MCMC-ABC) methodology [26]. Since the focus of this paper is on detection of the transmitted symbols, we will integrate out the nuisance channel parameters. We also develop two novel alternative solutions for comparison: an auxiliary variable MCMC (MCMC-AV) approach and a suboptimal explicit search zero forcing (SES$\mathrm{ZF}$ ) approach. In the MCMC-AV approach the addition of auxiliary variables results in closed form expressions for the full conditional posterior distributions. We use this fact to develop the MCMC-AV sampler and demonstrate that this works well when small numbers of relay nodes are considered. The SES-ZF approach involves an approximation based on known summary statistics of the channel model and an explicit exhaustive search over the parameter space of code words. This performs well for relatively small numbers of relays and a high signal to noise ratio (SNR).

The paper is organised as follows: in Section II we introduce a stochastic system model for the wireless relay system and the associated Bayesian model. We discuss the intractability arising from these models when one considers arbitrary relay functions. Section III presents the likelihood-free methodology and the generic MCMC-ABC algorithm. In Section IV we present the proposed algorithm, namely maximum a posteriori sequence detection. In Section $\mathrm{V}$ we derive an alternative novel algorithm based on an auxiliary variable model for the joint problem of coherent detection and channel estimation for arbitrary relay functions. We contrast the performance of this approach with the performance of the MCMC-ABC based algorithm. Section VI presents a detector that is based on known summary statistics of the channel model and an explicit exhaustive search over the parameter space of code words. We shall demonstrate its performance, whilst acknowledging its flaws. Section VII presents results and analysis and conclusions are provided in Section VIII.

The following notation is used throughout: random variables are denoted by upper case letters and their realizations by lower case letters. In addition, bold will be used to denote a vector or matrix quantity, upper subscripts will refer to the relay node index and lower subscripts refer to the element of a vector or matrix. We define the following notation that shall be used throughout, $\mathbf{g}=g_{1: L}=\left(g^{(1)}, \cdots, g^{(L)}\right) ; \mathbf{g}^{[n]}$ refers to the state of the Markov chain at iteration $n ; \mathbf{g}_{i}^{[n]}$ refers to the $i$-th element of $\mathbf{g}^{[n]}$. We define the generic transition kernel for the Markov chain as $q\left(\theta^{*} \leftarrow \theta^{[n-1]}\right)$, which updates the Markov chain for the posterior parameters, $\theta$, from the $(n-1)$-th Markov chain step to the $n$-th via the proposal $q$.

\section{BAYESIAN SYSTEM MODEL AND MAP DETECTION}

In this section we introduce the system model and a Bayesian model for inference on the system model parameters. In our system model, the channels in the relay network are modelled stochastically, where we do not know a priori the realized channel coefficient values. Instead, we consider partial channel state information (CSI), in which we assume known statistics of the distribution of the channel coefficients.

\section{A. System model and assumptions}

Here we present the system model and associated assumptions. The system model is depicted in Fig. 1.

1) Assume a wireless relay network with a single source node, transmitting sequences of $K$ symbols denoted $\mathbf{s}=$ $s_{1: K}$, to a single destination via $L$ relay nodes.

2) The symbols in the sequence of $K$ symbols $\mathbf{s}$ are taken from a digital constellation with cardinality $M$.

3) There are $L$ relays which cannot receive and transmit on the same time slot and on the same frequency band. We thus consider a half duplex system model in which the data transmission is divided into two steps. In the first step, the source node broadcasts a code word $\mathbf{s} \in \Omega$ from the codebook to all the $L$ relay nodes. In the second step, the relay nodes then transmit their signals to the destination node on orthogonal non-interfering channels. We assume that all channels are independent with a coherence interval larger than the codeword length $K$.

4) Assume imperfect CSI in which noisy estimates of the channel model coefficients for each relay link are known. This is a standard assumption based on the fact that a training phase has been performed a priori. This involves an assumption regarding the channel coefficients as follows:

- From source to relay there are L i.i.d. channels parameterized by $\left\{H^{(l)} \sim F\left(\widehat{h}^{(l)}, \sigma_{h}^{2}\right)\right\}_{l=1}^{L}$, where $F(\cdot)$ is the distribution of the channel coefficients, $\widehat{h}^{(l)}$ is the estimated channels coefficient and $\sigma_{h}^{2}$ is the associated estimation error variance. 
- From relay to destination there are L i.i.d. channels parameterized by $\left\{G^{(l)} \sim F\left(\widehat{g}^{(l)}, \sigma_{g}^{2}\right)\right\}_{l=1}^{L}$, where $F(\cdot)$ is the distribution of the channel coefficients, $\widehat{g}^{(l)}$ is the estimated channels coefficient and $\sigma_{g}^{2}$ is the associated estimation error variance.

5) The received signal at the l-th relay is a random vector given by

$$
\mathbf{R}^{(l)}=\mathbf{S} H^{(l)}+\mathbf{W}^{(l)}, l \in\{1, \cdots, L\},
$$

where $H^{(l)}$ is the channel coefficient between the transmitter and the l-th relay, $\mathbf{S} \in \Omega_{M}$ is the transmitted code-word and $\mathbf{W}^{(l)}$ is the noise realization associated with the relay receiver.

6) The received signal at the destination is a random vector given by

$$
\mathbf{Y}^{(l)}=\mathbf{f}^{(l)}\left(\mathbf{R}^{(l)}\right) G^{(l)}+\mathbf{V}^{(l)}, l \in\{1, \cdots, L\},
$$

where $G^{(l)}$ is the channel coefficient between the l-th relay and the receiver,

$\mathbf{f}^{(l)}\left(\mathbf{r}^{(l)}\right) \triangleq\left[f^{(l)}\left(r_{1}^{(l)}\right), \ldots, f^{(l)}\left(r_{K}^{(l)}\right)\right]^{\top}$ is the memoryless relay processing function (with possibly different functions at each of the relays) and $\mathbf{V}^{(l)}$ is the noise realization associated with the relay receiver.

7) Conditional on $\left\{h^{(l)}, g^{(l)}\right\}_{l=1}^{L}$, we have that all received signals are corrupted by zero-mean additive white complex Gaussian noise. At the l-th relay the noise corresponding to the l-th transmitted symbol is denoted by random variable $W_{i}^{(l)} \sim \mathrm{eN}\left(0, \sigma_{w}^{2}\right)$. At the receiver this is denoted by random variable $V_{i}^{(l)} \sim \operatorname{eN}\left(0, \sigma_{v}^{2}\right)$. Additionally, we assume the following properties:

$\mathbb{E}\left[W_{i}^{(l)} \bar{W}_{j}^{(m)}\right]=\mathbb{E}\left[V_{i}^{(l)} \bar{V}_{j}^{(m)}\right]=\mathbb{E}\left[W_{i}^{(l)} \bar{V}_{j}^{(m)}\right]=0$, $\forall i, j \in\{1, \ldots, K\}, \forall l, m \in\{1, \ldots, L\}, i \neq j, l \neq m$, where $\bar{W}_{j}$ denotes the complex conjugate of $W_{j}$.

\section{B. Prior specification and posterior}

Here we present the relevant aspects of the Bayesian model and associated assumptions. In order to construct a Bayesian model we need to specify the likelihood $p(\mathbf{y} \mid \mathbf{s}, \mathbf{h}, \mathbf{g})$, and the priors for the parameters $\mathbf{s}, \mathbf{h}, \mathbf{g}$ which are combined under Bayes theorem to obtain a posterior $p(\mathbf{s}, \mathbf{g}, \mathbf{h} \mid \mathbf{y})$. We begin by specifying the prior models for the sequence of symbols and the unknown channel coefficients. At this stage we note that in terms of system capacity it is only beneficial to transmit a sequence of symbols if it aids detection. This is achieved by having correlation in the transmitted symbol sequence $s_{1: K}$. We assume that since this is part of the system design, the prior structure for $p\left(s_{1: K}\right)$ will be known and reflect this information.

1) Under the Bayesian model, the symbol sequence is treated as a random vector $\mathbf{S}=S_{1: K}$. The prior for the random symbols sequence (code word) $S_{1: K}$ is defined on a discrete support denoted $\Omega$ with $|\Omega|=$ $M^{K}$ elements and probability mass function denoted by $p\left(s_{1: K}\right)$.
2) The assumption of imperfect CSI is treated under a Bayesian paradigm by formulating priors for the channel coefficients as follows:

- From source to relay there are L i.i.d. channels parameterized by $\left\{H^{(l)} \sim \operatorname{eN}\left(\widehat{h}^{(l)}, \sigma_{h}^{2}\right)\right\}_{l=1}^{L}$, where $\widehat{h}^{(l)}$ is the estimated channels coefficient and $\sigma_{h}^{2}$ the associated estimation error variance.

- From relay to destination there are L i.i.d. channels parameterized by $\left\{G^{(l)} \sim \operatorname{eN}\left(\widehat{g}^{(l)}, \sigma_{g}^{2}\right)\right\}_{l=1}^{L}$, where $\widehat{g}^{(l)}$ is the restituted channels coefficient and $\sigma_{g}^{2}$ the associated estimation error variance.

\section{Inference and MAP sequence detection}

Since the primary concern in designing a relay network system is on SER versus SNR, our goal is oriented towards detection of transmitted symbols and not the associated problem of channel estimation. We will focus on an approach which samples $S_{1: K}, \mathbf{G}=G_{1: L}, \mathbf{H}=H_{1: L}$ jointly from the target posterior distribution, since our model also considers the channels to be stochastic and unknown.

In particular we consider the maximum a posteriori (MAP) sequence detector at the destination node. Therefore the goal is to design a MAP detection scheme for $\mathbf{s}$ at the destination, based on the received signals $\left\{\mathbf{y}^{(l)}\right\}_{l=1}^{L}$, the noisy channels estimates as given by the partial CSI $\left\{\widehat{h}^{(l)}\right\}_{l=1}^{L},\left\{\widehat{g}^{(l)}\right\}_{l=1}^{L}$, $\sigma_{h}^{2}, \sigma_{g}^{2}$, and the noise variances, $\sigma_{w}^{2}$ and $\sigma_{v}^{2}$.

Since the channels are mutually independent, the received signals $\left\{\mathbf{r}^{(l)}\right\}_{l=1}^{L}$ and $\left\{\mathbf{y}^{(l)}\right\}_{l=1}^{L}$ are conditionally independent given $\mathbf{s}, \mathbf{g}, \mathbf{h}$. Thus, the MAP decision rule, after marginalizing out the unknown channel coefficients, is given by

$$
\begin{aligned}
\widehat{\mathbf{s}} & =\underset{\mathbf{s} \in \Omega}{\arg \max } p(\mathbf{s} \mid \mathbf{y}) \\
& =\underset{\mathbf{s} \in \Omega}{\arg \max } \prod_{l=1}^{L} \iint p\left(\mathbf{s}, g^{(l)}, h^{(l)} \mid \mathbf{y}^{(l)}\right) d \mathbf{h} d \mathbf{g} \\
& =\underset{\mathbf{s} \in \Omega}{\arg \max } \prod_{l=1}^{L} \iint p\left(\mathbf{y}^{(l)} \mid \mathbf{s}, h^{(l)}, g^{(l)}\right) p(\mathbf{s}) p(\mathbf{g}) p(\mathbf{h}) d \mathbf{h} d \mathbf{g} .
\end{aligned}
$$

Therefore, in order to perform detection of the transmitted symbols, we need to evaluate the likelihood function of the model. In the next section we will demonstrate that the likelihood function in our model is intractable and as a result we develop the likelihood-free based methodology and associated MCMC-ABC algorithm to perform the MAP detection.

\section{Evaluation of the likelihood function}

The likelihood model $p\left(\mathbf{y}^{(l)} \mid \mathbf{s}, h^{(l)}, g^{(l)}\right)$ for this relay system is in general computationally intractable as we now show. There are two potential difficulties that arise when dealing with non-linear relay functions. The first relates to finding the distribution of the signal transmitted from each relay to the destination. This involves finding the density of the random vector $\mathbf{f}^{(l)}\left(\mathbf{S} H^{(l)}+\mathbf{W}^{(l)}\right) G^{(l)}$ conditional on realizations $\mathbf{S}=\mathbf{s}, \mathbf{H}=\mathbf{h}, \mathbf{G}=\mathbf{g}$. This is not always possible for a general non-linear multivariate function $\mathbf{f}^{(l)}$. Conditional 
on $\mathbf{S}=\mathbf{s}, \mathbf{H}=\mathbf{h}, \mathbf{G}=\mathbf{g}$, we know the distribution of $\mathbf{R}^{(l)} \mid \mathbf{s}, \mathbf{g}, \mathbf{h}$,

$$
\begin{aligned}
& p_{\mathbf{R}}(\mathbf{r} \mid \mathbf{s}, \mathbf{g}, \mathbf{h}) \\
& =p\left(\mathbf{s} h^{(l)}+\mathbf{w}^{(l)} \mid \mathbf{s}, h^{(l)}, g^{(l)}\right)=\operatorname{eN}\left(\mathbf{s} h^{(l)}, \sigma_{w}^{2} \mathbf{I}\right) .
\end{aligned}
$$

However, finding the distribution of the random vector after the non-linear function is applied i.e. the distribution of $\widetilde{\mathbf{f}}\left(\mathbf{R}^{(l)}\right) \triangleq$ $\mathbf{f}\left(\mathbf{R}^{(l)}\right) G^{(l)}$ given $\mathbf{s}, h^{(l)}, g^{(l)}$, involves the following change of variable formula

$$
\begin{aligned}
& p\left(\widetilde{\mathbf{f}}\left(\mathbf{r}^{(l)}\right) \mid \mathbf{s}, h^{(l)}, g^{(l)}\right) \\
& =p_{\mathbf{R}}\left(\left(\widetilde{\mathbf{f}}^{(l)}\right)^{-1}\left(\mathbf{r}^{(l)}\right) \mid \mathbf{s}, h^{(l)}, g^{(l)}\right)\left|\frac{\partial \widetilde{\mathbf{f}}^{(l)}}{\partial \mathbf{r}^{(l)}}\right|^{-1},
\end{aligned}
$$

which can not always be written down analytically for arbitrary $\widetilde{\mathbf{f}}$. The second more serious complication is that even in cases where the density for the transmitted signal is known, one must then solve a $K$-fold convolution to obtain the likelihood:

$$
\begin{aligned}
& p\left(\mathbf{y}^{(l)} \mid \mathbf{s}, \mathbf{g}, \mathbf{h}\right)=p\left(\widetilde{\mathbf{f}}\left(\mathbf{r}^{(l)}\right) \mid \mathbf{s}, \mathbf{g}, \mathbf{h}\right) * p_{\mathbf{V}^{(l)}} \\
& =\int_{-\infty}^{\infty} \cdots \int_{-\infty}^{\infty} p(\widetilde{\mathbf{f}}(\mathbf{z} \mid \mathbf{s}, \mathbf{g}, \mathbf{h})) p_{\mathbf{V}^{(l)}}\left(\mathbf{y}^{(l)}-\mathbf{z}\right) d z_{1} \ldots d z_{K},
\end{aligned}
$$

where $*$ denotes the convolution operation. Typically this will be intractable to evaluate pointwise. However, in the most simplistic case of a linear relay function, that is, $\mathbf{f}^{(l)}(x)=x$, the likelihood can be obtained analytically as

$p\left(\mathbf{y}^{(l)} \mid \mathbf{s}, h^{(l)}, g^{(l)}\right)=\mathcal{e N}\left(\mathbf{s} h^{(l)} g^{(l)},\left(\left|g^{(l)}\right|^{2} \sigma_{w}^{2}+\sigma_{v}^{2}\right) \mathbf{I}\right)$,

where $\mathbf{I}$ is the identity matrix.

Hence, the resulting posterior distribution in (3) involves combining the likelihood given in (4) with the priors for the sequence of symbols and the channel coefficients.

\section{LIKELIHOOD-FREE METHODOLOGY}

In this section, we provide a concise background introduction on likelihood-free methodology, also known as approximate Bayesian computation, and the sampling algorithms utilised to obtain samples from the ABC posterior model, based on [18], [22], [26], [17]. Likelihood-free inference describes a suite of methods developed specifically for working with models in which the likelihood is computationally intractable. We consider the likelihood intractability to arise in the sense that we may not evaluate the likelihood pointwise, such as in (4). In particular, for the relay models considered, we can only obtain a general expression for the likelihood in terms of multivariate convolution integrals and hence we do not have an explicit closed form expression for which to evaluate the likelihood pointwise.

\section{ABC Methodology and MCMC-ABC:}

All ABC methodologies are based upon the observation that one may replace the problem of evaluating the intractable likelihood point-wise with the typically trivial exercise of simulating from the likelihood model. Simulation from the likelihood usually involves trivial generation of random variables from a known parametric distribution, followed by application of a transformation corresponding to the imposed physical model.

It is shown in [27] and further developed in [18] that the ABC method embeds an "intractable" target posterior distribution (resulting from the intractability of the likelihood function (4)), denoted by $p(\mathbf{s} \mid \mathbf{y})$, into an augmented posterior model,

$$
p(\mathbf{s}, \mathbf{x} \mid \mathbf{y}) \propto p(\mathbf{y} \mid \mathbf{x}, \mathbf{s} ; \epsilon) p(\mathbf{x} \mid \mathbf{s}) p(\mathbf{s})
$$

where $\mathbf{x} \in \mathbb{X}$ is an auxiliary vector on the same space as observations $\mathbf{y}$. In this augmented Bayesian model, the density $p(\mathbf{y} \mid \mathbf{x}, \mathbf{s} ; \epsilon)$ acts as a weight for the intractable posterior. It compares the observations $\mathbf{y}$ with the auxiliary ("synthetic data" $\mathbf{x}$ ) generated from the likelihood model, and determines whether or not they are within an $\epsilon$ tolerance of each other. Choices for this weighting, $p(\mathbf{y} \mid \mathbf{x}, \mathbf{s} ; \epsilon)$, will be explained below. For detailed justification for this framework see [18], [16] and [26]. In this paper we consider marginal sampler. Summarising the ideas in these papers, we note that the target marginal posterior $p(\mathbf{s} \mid \mathbf{y})$ can be represented in the $\mathrm{ABC}$ framework as follows

$$
\begin{aligned}
p(\mathbf{s} \mid \mathbf{y}) & \propto p(\mathbf{s}) p(\mathbf{y} \mid \mathbf{s}) \\
& =p(\mathbf{s}) \int_{\mathbb{X}} p(\mathbf{y} \mid \mathbf{x}, \mathbf{s} ; \epsilon) p(\mathbf{x} \mid \mathbf{s}) d \mathbf{x} \\
& =p(\mathbf{s}) \mathbb{E}_{p(\mathbf{x} \mid \mathbf{s})}[p(\mathbf{y} \mid \mathbf{x}, \mathbf{s} ; \epsilon)] \\
& \approx \frac{p(\mathbf{s})}{D} \sum_{d=1}^{D} p\left(\mathbf{y} \mid \mathbf{x}^{d}, \mathbf{s} ; \epsilon\right):=\widehat{p}(\mathbf{s} \mid \mathbf{y})
\end{aligned}
$$

Presenting the $\mathrm{ABC}$ approximate posterior in this way allows us to provide intuition for the ABC mechanism. To understand this, we note that the Monte Carlo approximation, denoted by $\widehat{p}(\mathbf{s} \mid \mathbf{y})$, of the integral with respect to the intractable likelihood, is approximated via $D$ draws of auxiliary variables $\mathbf{x}^{d}$ ("synthetic data") from $p(\mathbf{x} \mid \mathbf{s})$. Therefore, this demonstrates explicitly how one can replace the evaluation of the likelihood with simulation from the likelihood model. As discussed in [16], [17], the choice of weighting function can affect the Monte Carlo estimate. The function $p(\mathbf{y} \mid \mathbf{x}, \mathbf{s} ; \epsilon)$ is typically a standard smoothing kernel [28] with scale parameter $\epsilon$ which weights the intractable posterior with high values in regions when the observed data $\mathbf{y}$ and auxiliary date $\mathbf{x}$ are similar. For example, uniform kernels are commonplace in likelihood-free models (e.g. [26]), although alternatives such as Epanechnikov [15] and Gaussian kernels [17] provide improved efficiency.

In this paper we consider two popular choices for weighting functions, $p(\mathbf{y} \mid \mathbf{x}, \mathbf{s} ; \epsilon)$, which are easily interpreted as hard and soft decision functions [17]. The popular "Hard Decision" (HD) is given by

$$
p(\mathbf{y} \mid \mathbf{x}, \mathbf{s} ; \epsilon) \propto \begin{cases}1, & \text { if } \rho(\mathbf{y}, \mathbf{x}) \leq \epsilon \\ 0, & \text { otherwise }\end{cases}
$$

This makes a hard decision to reward those simulated auxiliary variables, $\mathbf{x}$, that are within an $\epsilon$-tolerance of the actual observed data, $\mathbf{y}$, as measured by distance metric $\rho$. Another example is a "Soft Decision" (SD) weighting function given 
by

$$
p(\mathbf{y} \mid \mathbf{x}, \mathbf{s} ; \epsilon) \propto \exp \left(-\frac{\rho(\mathbf{y}, \mathbf{x})}{\epsilon^{2}}\right) .
$$

An important practical difference between the HD and SD kernels is that, even though the weighting of the intractable posterior, obtained by the SD density, may be small, it will remain non-zero unlike the HD rule.

The choice of $\epsilon$ is therefore important for performances of the $\mathrm{ABC}$ methodology. If $\epsilon$ is too large, the approximation $\widehat{p}(\mathbf{s} \mid \mathbf{y})$ is poor; for example when $\epsilon \rightarrow \infty$ the resulting $\mathrm{ABC}$ posterior $\widehat{p}(\mathbf{s} \mid \mathbf{y})$ in (5) corresponds to the prior since the weighting function is uniform for all values of the posterior parameters. However, if $\epsilon$ is sufficiently small, $\widehat{p}(\mathbf{s} \mid \mathbf{y})$ is a good approximation of $p(\mathbf{s} \mid \mathbf{y})$. We note that there is no approximation when $\epsilon=0$. From a practical computational cost perspective, we will discuss why it is not feasible to set $\epsilon=0$ and instead we must settle for selecting an $\epsilon>0$ via a trade-off between accuracy and computation cost. There are several guidelines to specify a sequence of tolerances that one may follow to achieve a given $\epsilon$, see [29], [17], [21].

The following two sections provide specific background detail related to specifications of the weighting functions. We then finish this section by presenting the generic MCMC-ABC algorithm used to obtain samples from the marginal posterior $p(\mathbf{s} \mid \mathbf{y})$ in order to solve the MAP detection problem in (3).

\section{A. Data Summaries}

We note that when the total number of observations is large, then comparing directly the simulated data $\mathrm{x}$ to the observed data $\mathbf{y}$ in the weighting function is not computationally efficient. Therefore, one considers dimension reduction. The standard approach to dimension reduction is to compare summary statistics, $\mathbb{T}(\mathbf{y})$, which should summarise the information present in the data. The summary statistic $\mathbb{T}(\mathbf{y})$ is called a sufficient statistic for $\mathbf{y}$ if and only if $p(\mathbf{s} \mid \mathbf{y}) \propto p(\mathbf{s} \mid \mathbb{T}(\mathbf{y}))$, i.e., according to the Neayman-Fisher factorisation theorem, we can replace the conditional distribution of $\mathbf{y}$ given the parameters with the conditional distribution summary statistics given the parameters and a constant with respect to the parameters. If a sufficient statistic is available, then using the summary statistics is essentially the same as using the whole data. Therefore, as $\epsilon \rightarrow 0$, the $\mathrm{ABC}$ approximation $\widehat{p}(\mathbf{s} \mid \mathbb{T}(\mathbf{y})) \rightarrow p(\mathbf{s} \mid \mathbf{y}))$. However, in most real practical models, sufficient statistics are unknown and one must make alternative choices.

\section{B. Distance Metrics}

Having obtained summary statistic vectors $\mathbb{T}(\mathbf{y})$ and $\mathbb{T}(\mathbf{x})$, likelihood-free methodology then measures the distance between these vectors using a distance metric, denoted generically by $\rho(\mathbb{T}(\mathbf{y}), \mathbb{T}(\mathbf{x}))$. The most popular example involves the basic Euclidean distance metric which sums up the squared error between each summary statistic as follows:

$$
\rho(\mathbb{T}(\mathbf{y}), \mathbb{T}(\mathbf{x}))=\sum_{i}^{\operatorname{dim}(T)}\left(\mathbb{T}_{i}(\mathbf{y})-\mathbb{T}_{i}(\mathbf{x})\right)^{2} .
$$

Recently more advanced choices have been proposed and their impact on the methodology has been assessed [17]. These include: scaled Euclidean distance given by the square root of

$$
\rho(\mathbb{T}(\mathbf{y}), \mathbb{T}(\mathbf{x}))=\sum_{i}^{\operatorname{dim}(T)} \boldsymbol{\Lambda}_{i}\left(\mathbb{T}_{i}(\mathbf{y})-\mathbb{T}_{i}(\mathbf{x})\right)^{2} ;
$$

Mahalanobis distance:

$$
\rho(\mathbb{T}(\mathbf{y}), \mathbb{T}(\mathbf{x}))=(\mathbb{T}(\mathbf{y})-\mathbb{T}(\mathbf{x})) \Sigma^{-1}(\mathbb{T}(\mathbf{y})-\mathbb{T}(\mathbf{x}))^{T}
$$

$L^{p}$ norm:

$$
\rho(\mathbb{T}(\mathbf{y}), \mathbb{T}(\mathbf{x}))=\sum_{i}^{\operatorname{dim}(T)}\left[\left|\mathbb{T}_{i}(\mathbf{y})-\mathbb{T}_{i}(\mathbf{x})\right|^{p}\right]^{1 / p} ;
$$

and city block distance:

$$
\rho(\mathbb{T}(\mathbf{y}), \mathbb{T}(\mathbf{x}))=\sum_{i}^{\operatorname{dim}(T)}\left|\mathbb{T}_{i}(\mathbf{y})-\mathbb{T}_{i}(\mathbf{x})\right| .
$$

In particular, we note that distance metrics which include information regarding correlation between elements of each summary statistic vector, result in improved estimates of the marginal posterior.

\section{MCMC-ABC Samplers}

MCMC-based likelihood-free samplers were introduced to avoid the basic rejection sampling algorithms (see for example [30]) which are inefficient when the posterior and prior are sufficiently different [26]. Therefore, an MCMC approach that is based on the $\mathrm{ABC}$ methodology has been devised [26]. The generic MCMC-ABC sampler is presented in Algorithm 1.

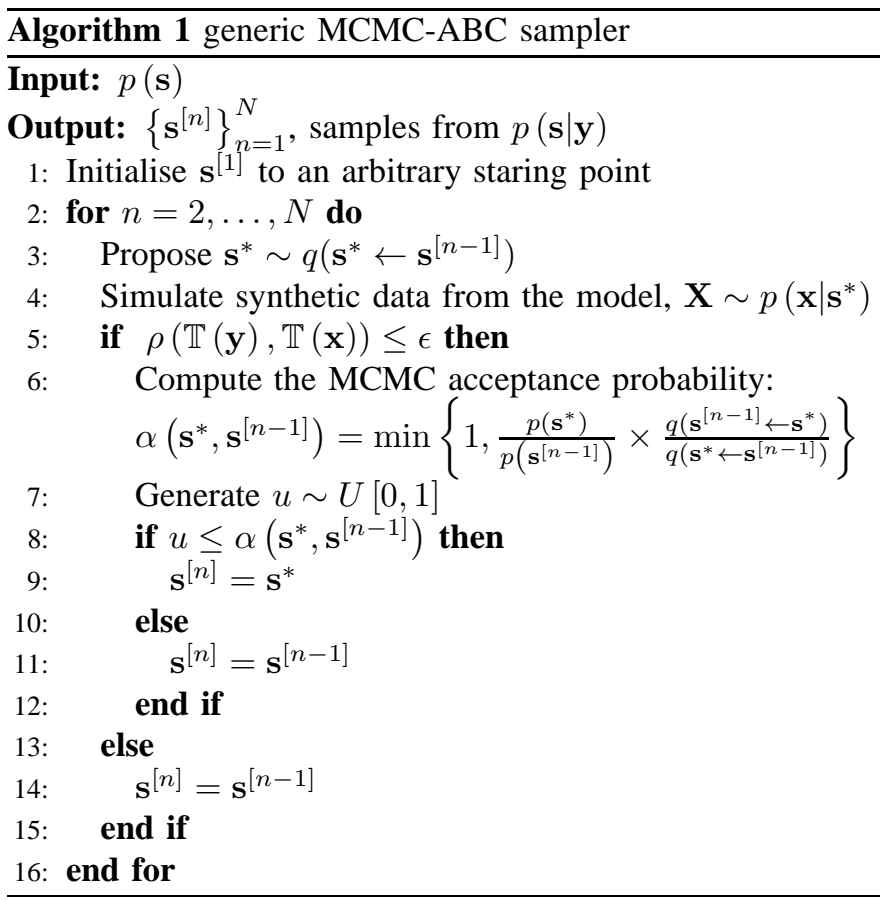

In the next section we present details of choices that must be made when constructing a likelihood-free inference model. 


\section{MCMC-ABC BASED DETECTOR}

We now relate the MCMC-ABC generic sampler to the specific problem presented in this paper. As mentioned previously, the $\mathrm{ABC}$ method we consider embeds an intractable target posterior distribution, in our case denoted by $p\left(s_{1: K}, h_{1: L}, g_{1: L} \mid \mathbf{y}\right)$, into a general augmented model

$$
\begin{aligned}
& p\left(s_{1: K}, h_{1: L}, g_{1: L}, \mathbf{x} \mid \mathbf{y}\right) \propto p\left(\mathbf{y} \mid \mathbf{x}, s_{1: K}, h_{1: L}, g_{1: L}\right) \\
& p\left(\mathbf{x} \mid s_{1: K}, h_{1: L}, g_{1: L}\right) p\left(s_{1: K}\right) p\left(h_{1: L}\right) p\left(g_{1: L}\right),
\end{aligned}
$$

where $\mathbf{x}$ is an auxiliary vector on the same space as $\mathbf{y}$. In this paper we make the standard $\mathrm{ABC}$ assumption, for the weighting function, $p\left(\mathbf{y} \mid \mathbf{x}, s_{1: K}, h_{1: L}, g_{1: L}\right)=p(\mathbf{y} \mid \mathbf{x})$, see [21].

Hence, in the $\mathrm{ABC}$ context, we obtain a general approximation to the intractable full posterior, denoted by $\widehat{p}\left(s_{1: K}, h_{1: L}, g_{1: L} \mid \mathbf{y}\right)$. We are interested in the marginal target posterior, $p\left(s_{1: K} \mid \mathbf{y}\right)$, which, in the $\mathrm{ABC}$ framework is approximated by

$$
\begin{aligned}
& p\left(s_{1: K} \mid \mathbf{y}\right) \\
& \propto \iiint p\left(\mathbf{y} \mid \mathbf{x}, s_{1: K}, h_{1: L}, g_{1: L} ; \epsilon\right) p\left(\mathbf{x} \mid s_{1: K}, h_{1: L}, g_{1: L}\right) p\left(s_{1: K}\right) \\
& p\left(h_{1: L}\right) p\left(g_{1: L}\right) d \mathbf{h} d \mathbf{g} d \mathbf{x} \\
& \approx p\left(s_{1: K}\right) \sum_{n=1}^{N} \sum_{d=1}^{D} p\left(\mathbf{y} \mid \mathbf{x}^{d,[n]}, s_{1: K}, h_{1: L}^{[n]}, g_{1: L}^{[n]} ; \epsilon\right) p\left(h_{1: L}^{[n]}\right) p\left(g_{1: L}^{[n]}\right) \\
& :=\widehat{p}\left(s_{1: K} \mid \mathbf{y}\right) \text {, }
\end{aligned}
$$

where $\mathrm{x}^{d,[n]}$ represents the $d$-th realisation at the $n$-th step of the Markov chain. In this paper we are interested in the marginal posterior $\mathrm{ABC}$ approximation $\widehat{p}\left(s_{1: K} \mid \mathbf{y}\right)$, as we wish to formulate the MAP detector for the symbols.

Next we present the resulting MCMC-ABC algorithm to perform MAP detection of a sequence of transmitted symbols. In particular our MCMC sampler is comprised of a random scan Metropolis-Hastings (MH) within Gibbs sampler [31]. The Gibbs sampler is a special case of the MH algorithm. It is typically used when one wishes to update sub-blocks of the posterior parameters at each iteration of the Markov chain. This is especially beneficial when the full conditional posterior distributions for each sub-block can be expressed in closed form expressions and sampled. In this case the transition kernel for the Markov chain on the full set of posterior parameters becomes a product of the full conditional posterior densities, resulting in acceptance probability of one, see [32]. If however, one cannot sample easily from any of the conditional posterior densities, an $\mathrm{MH}$ algorithm is used to produce a sample. This algorithm is referred to as a Metropolis-Hastings within Gibbs sampler.

Algorithm 2 presents the details of the sampler, where we use the compact notation $\Theta=(\mathbf{S}, \mathbf{G}, \mathbf{H})$. In order to utilise the Metropolis-Hastings algorithm, we now specify the Markov chain transition kernels for $\mathbf{S}, \mathbf{G}, \mathbf{H}$, otherwise known as the proposal distributions for the sampler. We design the sampler to update a single component of the posterior parameters, at every iteration of the algorithm, as it allows us to design proposals that insure a reasonable acceptance probability in the Markov chain rejection step. The transition kernels, denoted generically by $q\left(\theta^{*} \leftarrow \theta^{[n-1]}\right)$, which updates the Markov

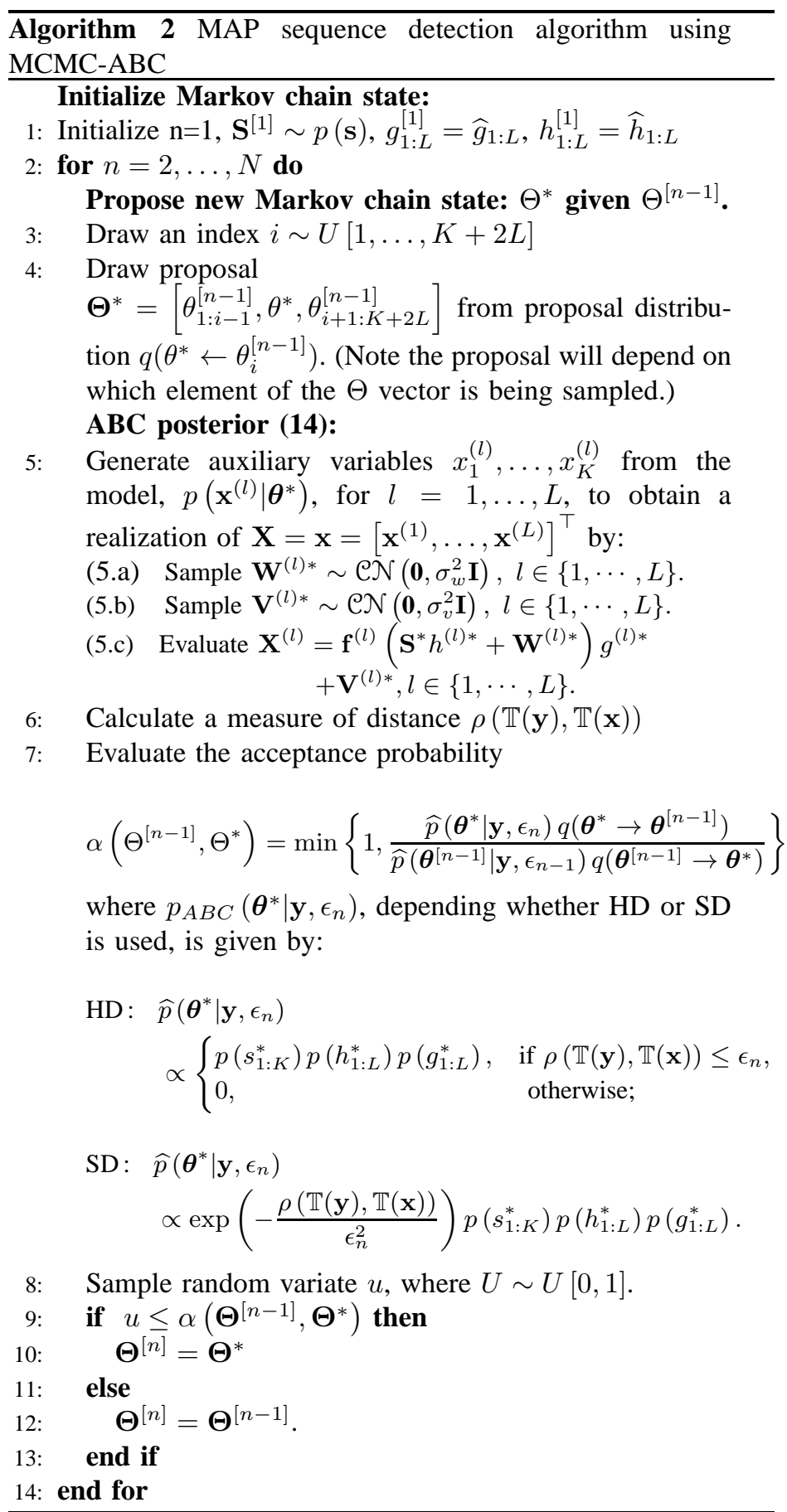

chain for the posterior parameters, $\theta$, from the $(n-1)$-th Markov chain step to the $n$-th via the proposal $q$. In this case we decompose $q$ into two components: the first, denoted by $q(i)$, specifies the transition probability mass function for sampling a proposed element of the posterior parameter vector to be updated; and the second, denoted by $q\left(\theta_{i} \mid \theta_{i}^{[n-1]}\right)$, specifies the probability density for proposing a new Markov chain state for element $\theta_{i}$ conditional on its previous state at iteration $n-1$. The specific transition kernels we specified for our algorithm are given by:

- Transition kernel for $\mathbf{S}_{1: K}$ : draw a proposal $\mathbf{S}_{1: K}^{*}$ from 
distribution

$$
\begin{aligned}
q\left(\mathbf{s}_{1: K}^{*} \leftarrow \mathbf{s}_{1: K}^{[n-1]}\right) & =q(i) q\left(\mathbf{s}_{i} \mid \mathbf{s}_{i}^{[n-1]}\right) \\
& =\frac{1}{K} \frac{1}{\log _{2} M} \delta_{\mathbf{s}_{1: i-1}^{[n-1]}} \delta_{\mathbf{s}_{i+1: K}^{[n-1]}} .
\end{aligned}
$$

- Transition kernel for $\mathbf{G}_{i}$ : draw proposal $\mathbf{G}_{i}^{*}$ from distribution

$q\left(\mathbf{g}^{*} \leftarrow \mathbf{g}_{i}^{[n-1]}\right)=q(l) q\left(g_{i}^{(l)} \mid \mathbf{g}_{i}^{[n-1]}\right)=\frac{1}{L} \mathcal{C N}\left(\mathbf{g}_{i}^{[n-1]}, \sigma_{g_{-} r w}^{2}\right)$

- Transition kernel for $\mathbf{H}_{i}$ : draw proposal $\mathbf{H}_{i}^{*}$ from distribution

$q\left(\mathbf{h}^{*} \leftarrow \mathbf{h}_{i}^{[n-1]}\right)=q(l) q\left(h_{i}^{(l)} \mid \mathbf{h}_{i}^{[n-1]}\right)=\frac{1}{L} \mathcal{C N}\left(\mathbf{h}_{i}^{[n-1]}, \sigma_{h_{-} r w}^{2}\right)$,

where $\delta_{\phi}$ denotes a dirac mass on location $\phi$, and $q(i)$ and $q(l)$ respectively denote the uniform probabilities of choosing indices $i \in\{1, \ldots, K\}$ and $l \in\{1, \ldots, L\}$, and $\sigma_{g_{-} r w}^{2}, \sigma_{h \_r w}^{2}$ represent the transition kernels variance. Thus, in every iteration we update a single component of the symbols $\mathbf{s}_{1: K}^{[n]}$, a single component of the transmitter-relay channels $\mathbf{g}_{i}^{[n]}$, and a single component of the relay-receiver channels $\mathbf{h}_{i}^{[n]}$.

We now present details about the choices made for the ABC components of the likelihood-free methodology.

\section{A. Observations and synthetic data}

The data $\mathbf{y}=y_{1: K}$ correspond to the observed sequence of symbols at the receiver. The generation of the synthetic data in the likelihood-free approach involves generating auxiliary variables $x_{1}^{(l)}, \ldots, x_{K}^{(l)}$ from the model, $p\left(\mathbf{x}^{(l)} \mid \mathbf{s}, \mathbf{g}, \mathbf{h}\right)$, for $l=$ $1, \ldots, L$, to obtain a realization $\mathbf{x}=\left[\mathbf{x}^{(1)}, \ldots, \mathbf{x}^{(L)}\right]^{\top}$. This is achieved under the following steps:

a. Sample $\mathbf{W}^{(l) *} \sim \mathcal{C N}\left(\mathbf{0}, \sigma_{w}^{2} \mathbf{I}\right)$.

b. Sample $\mathbf{V}^{(l) *} \sim \mathcal{C N}\left(\mathbf{0}, \sigma_{v}^{2} \mathbf{I}\right)$

c. Evaluate the system model in (2),

$$
\mathbf{X}^{(l)}=\mathbf{f}^{(l)}\left(\mathbf{S}^{*} h^{(l) *}+\mathbf{W}^{(l) *}\right) g^{(l) *}+\mathbf{V}^{(l) *}, l \in\{1, \cdots, L\} .
$$

\section{B. Summary statistics}

As discussed in Section III-A, summary statistics $\mathbb{T}(\cdot)$ are used in the comparison between the synthetic data and the observed data via the weighting function. There are many possible choices of summary statistic. Most critically, we want the summary statistics to be as close to sufficient statistics as possible whilst low in dimension. The simplest choice is to use $\mathbb{T}(\mathbf{y})=\mathbf{y}$ i.e. the complete dataset. This is optimal in the sense that it does not result in a loss of information from summarising the observations. The reason this choice is rarely used is that typically it will result in poor performance of the MCMC-ABC as discussed previously. To understand this, consider the HD rule weighting function in (6). In this case, even if the true MAP estimated model parameters were utilized to generate the synthetic data $\mathbf{x}$, it will still become improbable to realise a non-zero weight as $\epsilon \rightarrow 0$. This is made worse as the number of observations increases, through the curse of dimensionality. As a result, the acceptance probability in the MCMC-ABC algorithm would be zero for long periods, resulting in poor sampler efficiency. See [25] for a related discussion on chain mixing. We note that the exception to this rule is when a moderate tolerance $\epsilon$ and small number of observations are used.

A popular and practical alternative to utilizing the entire data set, is to use empirical quantile estimates of the data distribution. Here we adopt the vector of quantiles $\mathbb{T}(\mathbf{y})=$ $\left[\widehat{q}_{0.1}(\mathbf{y}), \ldots, \widehat{q}_{0.9}(\mathbf{y})\right]$, where $\widehat{q}_{\alpha}(\mathbf{y})$ denotes the $\alpha$-level empirical quantile of $\mathbf{y}$, see for example [20], [24].

\section{Distance metric}

For the distance metric $\rho(\mathbb{T}(\mathbf{y}), \mathbb{T}(\mathbf{x}))$, as a component of the weighting function, we use the Mahalanobis distance

$$
\rho(\mathbb{T}(\mathbf{y}), \mathbb{T}(\mathbf{x}))=[\mathbb{T}(\mathbf{y})-\mathbb{T}(\mathbf{x})]^{\top} \Sigma_{\mathbf{x}}^{-1}[\mathbb{T}(\mathbf{y})-\mathbb{T}(\mathbf{x})]
$$

where $\Sigma_{\mathbf{x}}$ is the covariance matrix of $\mathbb{T}(\mathbf{x})$. In Section VII we contrast this distance metric with scaled Euclidean distance, obtained by substituting $\operatorname{diag}\left(\Sigma_{\mathbf{x}}\right)$ for $\Sigma_{\mathbf{x}}$ in the above.

We estimate $\Sigma_{\mathbf{x}}$ as the sample covariance matrix of $\mathbb{T}(\mathbf{x})$, based on 2, 000 likelihood draws from $p\left(\mathbf{y} \mid \tilde{s}_{1: K}, \widehat{h}_{1: L}, \widehat{g}_{1: L}\right)$ where $\tilde{s}_{1: K}$ is a mode of the prior for the symbols and the channel coefficients are replaced with the partial CSI estimates.

We note that in principal, the choice of matrix $\Sigma_{\mathbf{x}}$ is immaterial, in the sense that in the limit as $\epsilon \rightarrow 0$, then $\widehat{p}\left(s_{1: K} \mid \mathbf{y}\right) \rightarrow p\left(s_{1: K} \mid \mathbf{y}\right)$ assuming sufficient statistics $\mathbb{T}(\mathbf{x})$. However, in practice, algorithm efficiency is directly affected by the choice of $\Sigma_{\mathbf{x}}$. We demonstrate this in Section VII.

\section{Weighting function}

For weighting function $p\left(\mathbf{y} \mid \mathbf{x}, s_{1: K}, h_{1: L}, g_{1: L}\right)=p(\mathbf{y} \mid \mathbf{x})$ we consider the HD weighting function in (6) and the SD weighting function in (7).

\section{E. Tolerance schedule}

With a HD weighting function, an MCMC-ABC algorithm can experience low acceptance probabilities for extended periods, particularly when the chain explores the tails of the posterior distribution (this is known as "sticking" c.f. [25]). In order to achieve improved chain convergence, we implement an annealed tolerance schedule during Markov chain burn-in, through $\epsilon_{n}=\max \left\{N-10 n, \epsilon^{\min }\right\}$, where $\epsilon_{n}$ is the tolerance at time $n$ in the Markov chain, $N$ is the overall number of Markov chain samples, and $\epsilon^{\text {min }}$ denotes the target tolerance of the sampler.

There is a trade-off between computational overheads (i.e. Markov chain acceptance rate) and the accuracy of the ABC posterior distribution relative to the true posterior. In this paper we determine $\epsilon^{\text {min }}$ via preliminary analysis of the Markov chain sampler mixing rates for a transition kernel with coefficient of variation set to one. In general practitioners will have a required precision in posterior estimates. This precision can be directly used to determine, for a given computational budget, a suitable tolerance $\epsilon^{\mathrm{min}}$. 


\section{F. Performance diagnostic}

Given the above mixing issues, one should carefully monitor convergence diagnostics of the resulting Markov chain for a given tolerance schedule. For a Markov chain of length $N$, the performance diagnostic we consider is the autocorrelation evaluated on $\tilde{N}=N-N_{b}$ post-convergence samples after an initial burn-in period $N_{b}$. Denoting by $\left\{\theta_{i}^{[n]}\right\}_{n=1: \tilde{N}}$ the Markov chain of the $i$-th parameter after burn-in, we define the autocorrelation estimate at lag $\tau$ by

$\widehat{A C F}\left(\theta_{i}, \tau\right)=\frac{1}{(\tilde{N}-\tau) \hat{\sigma}\left(\theta_{i}\right)} \sum_{n=1}^{\tilde{N}-\tau}\left[\theta_{i}^{[n]}-\widehat{\mu}\left(\theta_{i}\right)\right]\left[\theta_{i}^{[n+\tau]}-\widehat{\mu}\left(\theta_{i}\right)\right]$,

where $\widehat{\mu}\left(\theta_{i}\right)$ and $\hat{\sigma}\left(\theta_{i}\right)$ are the estimated mean and standard deviation of $\theta_{i}$.

\section{AuXiliary VARIABle MCMC APPROACH}

In this section we demonstrate an alternative solution to the previously presented MCMC-ABC detector. We will show that at the expense of increasing the dimension of the parameter vector, one can develop a standard MCMC algorithm without the requirement of the ABC methodology. We augment the parameter vector with the unknown noise realizations at each relay, $\mathbf{w}_{1: K}$, to obtain a new parameter vector $\left(\mathbf{s}_{1: K}, g_{1: L}, h_{1: L}, \mathbf{w}_{1: K}\right)$. The resulting posterior distribution $p\left(\mathbf{s}_{1: K}, g_{1: L}, h_{1: L}, \mathbf{w}_{1: K} \mid \mathbf{y}\right)$ may then be decomposed into the full conditional distributions:

$p\left(\mathbf{s}_{1: K} \mid g_{1: L}, h_{1: L}, \mathbf{w}_{1: K}, \mathbf{y}\right) \propto p\left(\mathbf{y} \mid \mathbf{s}_{1: K}, g_{1: L}, h_{1: L}, \mathbf{w}_{1: K}\right) p\left(\mathbf{s}_{1: K}\right)$,

$p\left(g_{1: L} \mid \mathbf{s}_{1: K}, h_{1: L}, \mathbf{w}_{1: K}, \mathbf{y}\right) \propto p\left(\mathbf{y} \mid \mathbf{s}_{1: K}, g_{1: L}, h_{1: L}, \mathbf{w}_{1: K}\right) p\left(g_{1: L}\right)$,

$p\left(h_{1: L} \mid \mathbf{s}_{1: K}, g_{1: L}, \mathbf{w}_{1: K}, \mathbf{y}\right) \propto p\left(\mathbf{y} \mid \mathbf{s}_{1: K}, g_{1: L}, h_{1: L}, \mathbf{w}_{1: K}\right) p\left(h_{1: L}\right)$,

$p\left(\mathbf{w}_{1: K} \mid \mathbf{s}_{1: K}, g_{1: L}, h_{1: L}, \mathbf{y}\right) \propto p\left(\mathbf{y} \mid \mathbf{s}_{1: K}, g_{1: L}, h_{1: L}, \mathbf{w}_{1: K}\right) p\left(\mathbf{w}_{1: K}\right)$

which form a block Gibbs sampling framework. Conditioning on the unknown noise random variables at the relays permits a simple closed form solution for the likelihood and results in tractable full conditional posterior distributions for (19a)(19d). In this case the likelihood is given by

$p\left(\mathbf{y} \mid \mathbf{s}_{1: K}, g_{1: L}, h_{1: L}, \mathbf{w}_{1: K}\right)=\prod_{l=1}^{L} p\left(\mathbf{y}^{(l)} \mid \mathbf{s}_{1: K}, g^{(l)}, h^{(l)}, \mathbf{w}^{(l)}\right)$

where

$p\left(\mathbf{y}^{(l)} \mid \mathbf{s}_{1: K}, g^{(l)}, h^{(l)}, \mathbf{w}^{(l)}\right)=\mathcal{C N}\left(\mathbf{f}^{(l)}\left(\mathbf{S} h^{(l)}+\mathbf{W}^{(l)}\right) g^{(l)}, \sigma_{v}^{2} \mathbf{I}\right)$

The resulting Metropolis-within Gibbs sampler for this block Gibbs framework is outlined in Algorithm 3, where we define the joint posterior parameter vector $\Theta=(\mathbf{S}, \mathbf{G}, \mathbf{H}, \mathbf{W})$. The Metropolis-Hastings proposals used to sample from each full conditional distribution were given by eqs. (15)-(17), and the additional proposal for the auxiliary variables, given by

- Transition kernel for $\mathbf{W}_{i}$ : draw proposal $\mathbf{W}_{i}^{*}$ from distribution

$$
\begin{aligned}
q\left(\mathbf{w}^{*} \leftarrow \mathbf{w}_{i}^{[n-1]}\right) & =q(l) q(i) q\left(w_{i}^{(l)} \mid \mathbf{w}_{i}^{[n-1]}\right) \\
& =\frac{1}{K L} \mathcal{C N}\left(\mathbf{w}_{i}^{[n-1]}, \sigma_{w \_r w}^{2}\right) .
\end{aligned}
$$

$\overline{\text { Algorithm } 3 \text { MAP sequence detection algorithm using AV- }}$ MCMC

Initialize Markov chain state:

1: Initialize $\mathrm{n}=1, \mathbf{S}^{[1]} \sim p(\mathbf{s}), g_{1: L}^{[1]}=\widehat{g}_{1: L}, h_{1: L}^{[1]}=\widehat{h}_{1: L}$, $\mathbf{W}^{(0)} \sim p(\mathbf{w})$

2: for $n=1, \ldots, N$ do

Propose new Markov chain state: $\Theta^{*}$ given $\Theta^{[n-1]}$.

3: $\quad$ Draw an index $i \sim U[1, \ldots, K+2 L+K L]$

4: $\quad$ Draw proposal $\Theta^{*}=\left[\theta_{1: i-1}^{[n-1]}, \theta^{*}, \theta_{i+1: K+2 L+K L}^{[n-1]}\right]$ from proposal distribution $q\left(\theta_{i}^{n-1]} \rightarrow \theta^{*}\right)$

(Note, the proposal will depend on which element of the $\Theta$ vector is being sampled.)

5: Evaluate the acceptance probability

$$
\alpha\left(\Theta^{[n-1]}, \Theta^{*}\right)=\min \left\{1, \frac{p\left(\boldsymbol{\theta}^{*} \mid \mathbf{y}\right) q\left(\boldsymbol{\theta}^{*} \rightarrow \boldsymbol{\theta}^{[n-1]}\right)}{p\left(\boldsymbol{\theta}^{[n-1]} \mid \mathbf{y}\right) q\left(\boldsymbol{\theta}^{[n-1]} \rightarrow \boldsymbol{\theta}^{*}\right)}\right\} .
$$

6: $\quad$ Sample random variate $u$, where $U \sim U[0,1]$.

7: $\quad$ if $u \leq \alpha\left(\boldsymbol{\Theta}^{[n-1]}, \boldsymbol{\Theta}^{*}\right)$ then

8: $\quad \boldsymbol{\Theta}^{[n]}=\boldsymbol{\Theta}^{*}$

9: $\quad$ else

10: $\quad \boldsymbol{\Theta}^{[n]}=\boldsymbol{\Theta}^{[n-1]}$.

11: end if

12: end for

The MCMC-AV approach presents an alternative to the likelihood-free Bayesian model sampler, and produces exact samples from the true posterior following chain convergence. While the MCMC-AV sampler still performs joint channel estimation and detection, the trade-off is that sampling the large number of extra parameters will typically result the need for longer Markov chains, to achieve the same performance as the $\mathrm{ABC}$ algorithm (in terms of joint estimation and detection performance). This is especially true in high dimensional problems, such as when the sequence of transmitted symbols $K$ is long and the number of relays $L$ present in the system is large, or when the posterior distribution of the additional auxiliary variables exhibits strong dependence.

\section{Alternative MAP DETECTORS AND LOWER BOUNDS}

One can define a suboptimal solution to the MAP detector, even with an intractable likelihood, involving a naive, highly computational algorithm based on a Zero Forcing (ZF) solution. The ZF solution is popular in simple system models where it can be efficient and performs well.

Under a ZF solution one conditions on some knowledge of the partial channel state information, and then perform an explicit search over the set of all possible symbol sequences. To our knowledge a ZF solution for MAP sequence detection in arbitrary non-linear relay systems has not been defined. Accordingly we define the ZF solution for MAP sequence detection as the solution which conditions on the mean of the noise at the relay nodes, and also uses the noisy channel estimates given by the partial CSI information, to reduce the detection search space. 


\section{A. Sub-optimal exhaustive search Zero Forcing approach}

In this approach we condition on the mean of the noise $\mathbf{W}^{(l)}=\mathbf{0}$, and use the partial CSI estimates of channels coefficients, $\left\{\widehat{h}^{(l)}, \widehat{g}^{(l)}\right\}_{l=1}^{L}$, to reduce the dimensionality of the MAP detector search space to just the symbol space $\Omega$. The SES-ZF-MAP sequence detector can be expressed as

$$
\begin{aligned}
\widehat{\mathbf{s}} & =\underset{\mathbf{s} \in \Omega}{\arg \max } \prod_{l=1}^{L} p\left(\mathbf{s} \mid \mathbf{y}^{(l)}, G^{(l)}=\widehat{g}^{(l)}, H^{(l)}=\widehat{h}^{(l)}, \mathbf{W}^{(l)}=\mathbf{0}\right) \\
& =\underset{\mathbf{s} \in \Omega}{\arg \max } \prod_{l=1}^{L} p\left(\mathbf{y}^{(l)} \mid \mathbf{s}, G^{(l)}=\widehat{g}^{(l)}, H^{(l)}=\widehat{h}^{(l)}, \mathbf{W}^{(l)}=\mathbf{0}\right) p(\mathbf{s}) .
\end{aligned}
$$

Thus, the likelihood model results in a complex Gaussian distribution for each relay channel, as follows

$$
\begin{aligned}
& p\left(\mathbf{y}^{(l)} \mid \mathbf{s}, G^{(l)}=\widehat{g}^{(l)}, H^{(l)}=\widehat{h}^{(l)}, \mathbf{W}^{(l)}=\mathbf{0}\right) \\
& =\operatorname{eN}\left(\mathbf{f}^{(l)}\left(\mathbf{s} \widehat{h}^{(l)}\right) \widehat{g}^{(l)}, \sigma_{v}^{2} \mathbf{I}\right) .
\end{aligned}
$$

As a result, the MAP detection can be solved exactly using an explicit search.

Note however that this approach to symbol detection also involves a very high computational cost, as one must evaluate the posterior distribution for all $M^{K}$ code words in $\Omega$. It is usual for communications systems to utilise $M$ as either 64ary PAM or 128-ary PAM and the number of symbols can be anything from $K=1$ to $K=20$ depending on the channel capacity budget for the designed network and the typical operating SNR level. Typically this explicit search is not feasible to perform. However, the sub-optimal ZF-MAP detector provides a comparison for the MCMC-ABC approach, which at low SNR should be a reasonable upper bound for the SER and for high SNR an approximate optimal solution.

The SES-ZF-MAP sequence detector can be highly suboptimal for low SNR values. This is trivial to see, since we are explicitly setting the noise realisations to zero when the variance the noise distribution is large. For the same reasoning, in high SNR values, the ZF approach becomes close to optimal.

\section{B. Lower bound MAP detector performance}

We denote the theoretical lower bound for the MAP detector performance as the oracle MAP detector (OMAP). The OMAP detector involves conditioning on perfect oracular knowledge of the channels coefficients $\left\{h^{(l)}, g^{(l)}\right\}_{l=1}^{L}$ and of the realized noise sequence at each relay $\mathbf{W}^{(l)}$. This results in the likelihood model for each relay channel being complex Gaussian, resulting in an explicit solution for the MAP detector. Accordingly, the OMAP detector provides the lower bound for the SER performance. The OMAP detector is expressed as

$$
\widehat{\mathbf{s}}=\underset{\mathbf{s} \in \Omega}{\arg \max } \prod_{l=1}^{L} p\left(\mathbf{s} \mid \mathbf{y}^{(l)}, \boldsymbol{\Pi}\right)=\underset{\mathbf{s} \in \Omega}{\arg \max } \prod_{l=1}^{L} p\left(\mathbf{y}^{(l)} \mid \mathbf{s}, \boldsymbol{\Pi}\right) p(\mathbf{s}),
$$

where $\boldsymbol{\Pi}:=\left(G^{(l)}=g^{(l)}, H^{(l)}=h^{(l)}, \mathbf{W}^{(l)}=\mathbf{W}^{(l)}\right)$. In this case, the likelihood model results in a complex Gaus- sian distribution for each relay channel, as follows

$$
\begin{aligned}
& p\left(\mathbf{y}^{(l)} \mid \mathbf{s}, G^{(l)}=g^{(l)}, H^{(l)}=h^{(l)}, \mathbf{W}^{(l)}=\mathbf{W}^{(l)}\right) \\
& =\operatorname{eN}\left(\mathbf{f}^{(l)}\left(\mathbf{s} h^{(l)}+\mathbf{W}^{(l)}\right) g^{(l)}, \sigma_{v}^{2} \mathbf{I}\right) .
\end{aligned}
$$

However, clearly this is impossible to evaluate in a real system, since oracular knowledge is not available.

\section{RESUlts}

We compare the performance of joint channel estimation and detection under the $\mathrm{ABC}$ relay methodology versus the auxiliary MCMC approach for different model configurations. Additionally, we compare the detection performance of the $\mathrm{ABC}$ relay methodology, the auxiliary MCMC approach, the optimal oracle MAP sequence detector and the SES-ZF MAP sequence detector. Each presented Monte Carlo sampler has a burn-in of 5,000 iterations followed by a further 15,000 recorded iterations $(N=20,000)$. Random walk proposal variances for each of the parameters were tuned off-line to ensure the average acceptance probability (post burn-in) was in the range 0.3 to 0.5 .

The following specifications for the relay system model are used for all the simulations performed: the symbols are taken from a constellation which is 4-PAM at constellation points in $\{-3,-1,1,3\}$; each sequence contains $K=2$ symbols; the prior for the sequence of symbols is $p\left(\left(s_{1}, s_{2}\right)=[1,1]\right)=$ $p\left(\left(s_{1}, s_{2}\right)=[-1,1]\right)=0.3$ otherwise, all other are equiprobable; the partial CSI is given by $\sigma_{g}^{2}=\sigma_{h}^{2}=0.1$; the nonlinear relay function is given by $f(\cdot)=\tanh (\cdot)$. These system parameters were utilised as they allow us to perform the zero forcing solution, without a prohibitive computational burden.

\section{A. Analysis of mixing and convergence of $M C M C-A B C$}

We analyze the impact that the $\mathrm{ABC}$ tolerance level $\epsilon^{\mathrm{min}}$ has on estimation performance of channel coefficients and the mixing properties of the Markov chain for the MCMC$\mathrm{ABC}$ algorithm. The study involves joint estimation of channel coefficients and transmitted symbols at an SNR level of $15 \mathrm{~dB}$, with $L=5$ relays present in the system. We adopt a scaled Euclidean distance metric with the HD weighting function (6) and empirically monitor the mixing of the Markov chain through the autocorrelation function (ACF).

In Fig. 2 we present a study of the ACF of the Markov chains for the channel estimations of $G_{1}$ and $H_{1}$ as a function of the tolerance $\epsilon$, and the associated estimated marginal posterior distributions $p\left(g_{1} \mid \mathbf{Y}\right)$ and $p\left(h_{1} \mid \mathbf{Y}\right)$. For large $\epsilon$ the Markov chain mixes over the posterior support efficiently, since when the tolerance is large, the HD weighting function and therefore the acceptance probability, will regularly be non-zero. In addition, with a large tolerance the posterior almost exactly recovers the prior given by the partial CSI. This is expected since a large tolerance results in a weak contribution from the likelihood. As the tolerance $\epsilon$ decreases, the posterior distribution precision increases and there is a translation from the prior partial CSI channel estimates to 

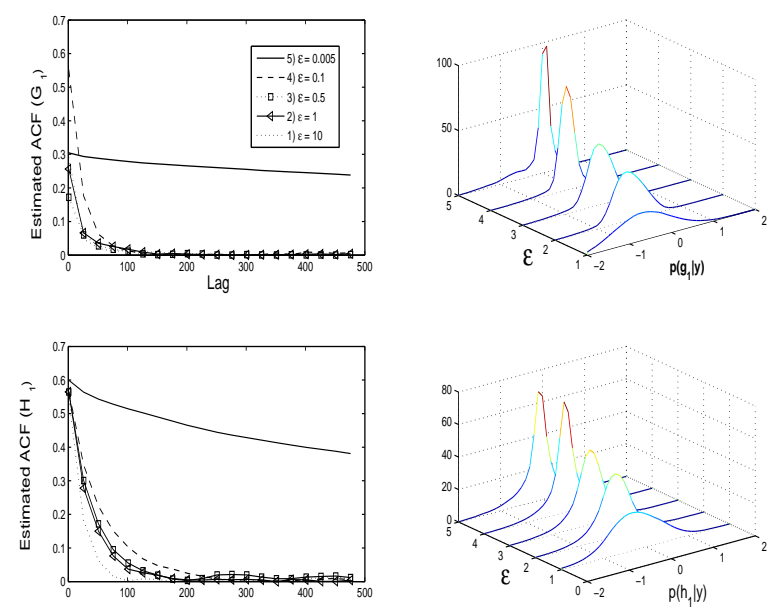

Fig. 2. Comparison of performance for MCMC-ABC with Hard Decision weighting and Scaled Euclidean distance metric. Subplots on the left of the image display how the estimated ACF changes as a function of tolerance level $\epsilon$ for the estimated channels of the relay system. Subplots on the right of the image display a sequence of smoothed marginal posterior distribution estimates for the first channel of the relay, as the tolerance decreases. Note, the indexing of each marginal distribution with labels $1,2, \ldots$ corresponds to the tolerance given in the legend on the left hand plots.

the posterior distribution over the true generated channel coefficients for the given frame of data communications.

It is evident that the mixing properties of the MCMC$\mathrm{ABC}$ algorithm are impacted by the choice of tolerance level. A decreasing tolerance results in more accurate posterior distribution, albeit at the price of slower chain mixing. Clearly, the ACF tail decay rate is significantly slower as the tolerance reduces.

Note, that although the results are not presented here, we also performed analysis for all aspects of Algorithm 1 under the setting in which the relay function is linear. We confirmed the MMSE estimates of the channel coefficients and the MAP sequence detector results were accurate for a range of SNR values. The results presented here are for a much more challenging setting in which the relay is highly non-linear, given by a hyperbolic tangent function.

\section{B. Analysis of ABC model specifications}

We now examine the effect of the distance metric and weighting function on the performance of the MCMC-ABC algorithm as a function of the tolerance. We consider HD and SD weighting functions with both Mahalanobis and scaled Euclidean distance metrics. We consider the estimated $\mathrm{ACF}$ of the Markov chains of each of the channel coefficients $G$ and $H$. The SNR level was set to $15 \mathrm{~dB}$ and $L=5$ relays were present. The results are presented for one channel; since all channels are i.i.d. this will be indicative of the performance of all channels.

For comparison, an equivalent $\mathrm{ABC}$ posterior precision should be obtained under each algorithm. Since, the weighting and distance functions are different, this will result in different

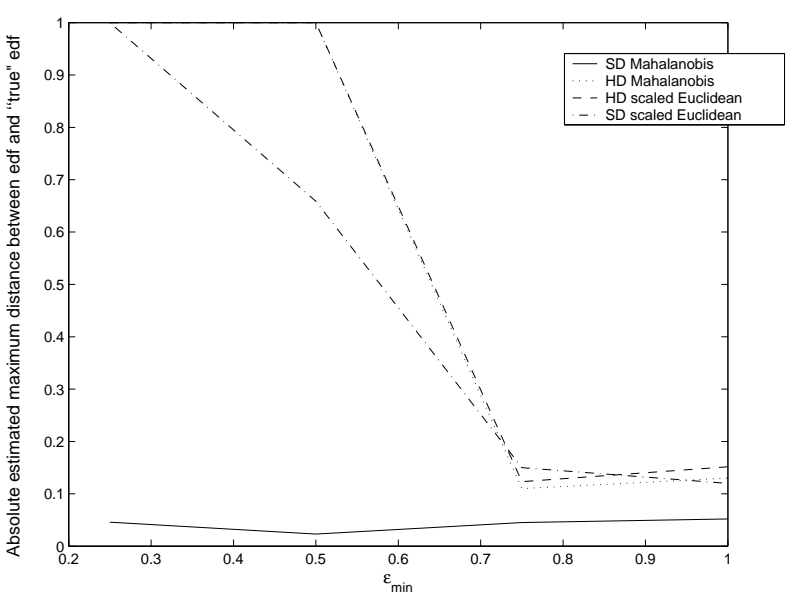

Fig. 3. Maximum distance between the EDF and the baseline "true" EDF for the first channel, estimated cdf for $G_{1}$, averaged over 20 independent data realisations.

$\epsilon^{\text {min }}$ values for each choice in the MCMC-ABC algorithm. As a result, analysis proceeded by first taking a minimum base epsilon value, $\epsilon_{b}=0.2$ and running the MCMC$\mathrm{ABC}$ with soft decision Gaussian weighting and Mahalanobis distance for 100,000 simulations. We ensured that the average acceptance rate was between $[0.1,0.3]$. This produced a "true" empirical distribution function (EDF) which we used as our baseline comparison estimate of the true cdf. Now, for a range of tolerance values $\epsilon_{i}=[0.25,0.5,0.75,1]$ we ran the MCMC-ABC algorithm with soft decision Gaussian weighting and Mahalanobis distance for 20,000 iterations, ensuring the average acceptance rate was in the interval $[0.1,0.3]$. This produced a set of random walk standard deviation $\sigma_{b(i)}$, one for each tolerance, that we used for the analysis in the remaining choices of the MCMC-ABC algorithms. For comparison purposes we recorded the estimated maximum error between the estimated EDF for each algorithm and the baseline "true" EDF. We repeated the simulations for each tolerance on 20 independent generated data sets.

In Fig. 3 we present the results of this analysis, which demonstrate that the algorithm producing the most accurate results utilised the soft decision and Mahalanobis distance. The worst performance involved the hard decision and scaled Euclidean distance. In this case, at low tolerances the average distance between the EDF and the baseline "true" EDF was a maximum, since the algorithm was not mixing. This demonstrates that such low tolerances under this setting of the MCMC-ABC algorithm will produce poor performance, relative to the soft decision with Mahalanobis distance.

\section{Comparisons of detector performance}

Finally, we present an analysis of the symbol error rate (SER) under the MCMC-ABC algorithm (with a SD weighting function and Mahalanobis distance), the MCMC-AV detector algorithm, and the SES-ZF and Oracle detectors. Specifically, we systematically study the SER as a function of the number of relays, $L \in\{1,2,5,10\}$ and the SNR $\in$ $\{0,5,10,15,20,25,30\}$. 

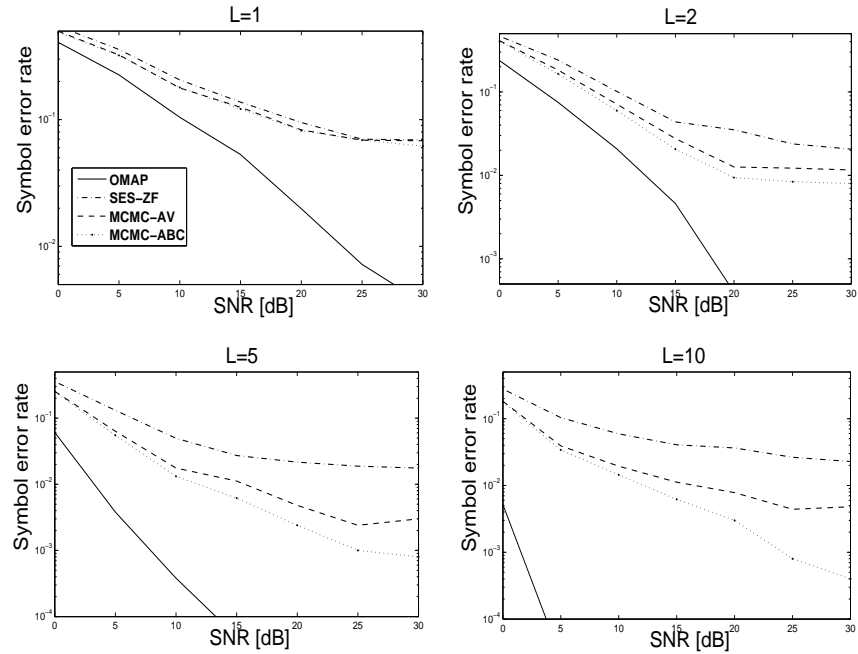

Fig. 4. SER performance of each of the proposed detector schemes as a function of the number of relay links, $L$. For each relay set up the SER is reported as a function of the SNR.

The results of this analysis are presented in Fig. 4. In summary these results demonstrate that under our proposed system model and detection algorithms, spatial diversity stemming from an increasing number of relays results in measurable improvements in the SER performance. For example Fig. 4 demonstrates that for $L=1$, there is an insignificant difference between the results obtained for algorithms MCMCABC, MCMC-AV and SES-ZF. However, as $L$ increases, SES$\mathrm{ZF}$ has the worst performance and degrades relative to the MCMC-based approaches, demonstrating the utility obtained by developing a more sophisticated detector algorithm. It is clear that the SES-ZF suffers from an error-floor effect: as the SNR increases the SER is almost constant for SNR values above $15 \mathrm{~dB}$.

Also in Fig. 4 the two MCMC-based approaches demonstrate comparable performance for small $L$. However as $L$ increases, in the high SNR region, the difference in performance between the MCMC-AV and MCMC-ABC algorithms increases. This could be due to the greater numbers of auxiliary parameters to be estimated in the auxiliary-based approach as $L$ increases. In particular we note that adding an additional relay introduces $K$ additional nuisance parameters into the auxiliary model posterior.

\section{CONCLUSIONS}

In this paper, we proposed a novel cooperative relay system model and then obtained novel detector algorithms. In particular, this involved an approximated-MAP sequence detector for a coherent multiple relay system, where the relay processing function is non-linear. Using the ABC methodology we were able to perform "likelihood free" inference on the parameters of interest. Simulation results validate the effectiveness of the proposed scheme. In addition to the ABC approach, we developed an alternative exact novel algorithm, MCMC-AV, based on auxiliary variables. Finally, we developed a sub-optimal zero forcing solution. We then studied the performance of each algorithm under different settings of our relay system model, including the size of the network and the noise level present. As a result of our findings, we recommend the use of the MCMC-ABC detector especially when there are many relays present at the network, or the number of symbols transmitted in each frame is large. In settings where the number of relays is moderate, one could consider using the MCMC-AV algorithm, as its performance was on par with the MCMC-ABC results and does not involve an $\mathrm{ABC}$ approximation.

Future research includes the design of detection algorithms for relay systems with partial CSI in which the relay system topology may contain multiple hops on a given channel, or the relay network topology may be unknown. This can include aspects such as an unknown number of relay channels or hops per relay channel.

\section{Acknowledgements}

The authors would also like to thank the reviewers for their thorough and thoughtful comments. GWP thanks Prof. Arnaud Doucet and Dr. Mark Briers for useful discussion and the Department of Mathematics and Statistics at UNSW (through an APA) and CMIS, CSIRO for financial support. This material was based upon work partially supported by the National Science Foundation under Grant DMS-0635449 to the Statistical and Applied Mathematical Sciences Institute, North Carolina, USA. Any opinions, findings, and conclusions or recommendations expressed in this material are those of the author(s) and do not necessarily reflect the views of the NSF. SAS and YF were supported by the Australian Research Council through the Discovery Project scheme (DP0664970 and DP1092805). IN and JY were supported by the Australian Research Council through the Discovery Project scheme (DP0667030).

\section{REFERENCES}

[1] A. Nosratinia, T. Hunter, and A. Hedayat, "Cooperative communication in wireless networks," IEEE Communications Magazine, vol. 42, no. 10, pp. 74-80, 2004.

[2] J. Laneman, D. Tse, and G. Wornell, "Cooperative diversity in wireless networks: Efficient protocols and outage behavior," Information Theory, IEEE Transactions on, vol. 50, no. 12, pp. 3062-3080, 2004.

[3] E. van der Meulen, "Three-terminal communication channels," $A d v$. Appl. Prob, vol. 3, no. 1, pp. 120-154, 1971.

[4] J. Laneman and G. Wornell, "Distributed space-time-coded protocols for exploiting cooperative diversity in wireless networks," Information Theory, IEEE Transactions on, vol. 49, no. 10, pp. 2415-2425, 2003.

[5] T. Cover and A. Gamal, "Capacity theorems for the relay channel," Information Theory, IEEE Transactions on, vol. 25, no. 5, pp. 572-584, 1979.

[6] D. Chen and J. Laneman, "Modulation and Demodulation for Cooperative Diversity in Wireless Systems," IEEE Transactions on Wireless Communications, vol. 5, no. 7, pp. 1785-1794, 2006.

[7] G. Kramer, M. Gastpar, and P. Gupta, "Cooperative Strategies and Capacity Theorems for Relay Channels," IEEE Transactions on Information Theory, vol. 51, no. 9, pp. 3037-3063, 2005.

[8] M. Khojastepour, A. Sabharwal, and B. Aazhang, "On the capacity of cheap relay networks," pp. 12-14, 2003.

[9] K. Gomadam and S. Jafar, "Optimal relay functionality for SNR maximization in memoryless relay networks," IEEE Journal on Selected Areas in Communications, vol. 25, no. 2, pp. 390-401, 2007.

[10] L. Ghabeli and M. Aref, "A new achievable rate and the capacity of some classes of multilevel relay network," EURASIP Journal on Wireless Communications and Networking, vol. 2008, p. 38, 2008.

[11] D. Crouse, C. Berger, S. Zhou, and P. Willett, "Optimal memoryless relays with noncoherent modulation," IEEE Trans. Signal Processing, vol. 56 , no. 12 , p. 1,2008 . 
[12] S. Yao, M. Khormuji, and M. Skoglund, "Sawtooth relaying," IEEE Communication Letters, vol. 12, no. 9, pp. 612-615, 2008.

[13] T. Cui, T. Ho, and J. Kliewer, "Some results on relay strategies for memoryless two-way relay channels," Proc. of Information Theory and Applications Workshop, pp. 158-164, 2008.

[14] O. Ratmann, O. Jørgensen, T. Hinkley, M. Stumpf, S. Richardson, and C. Wiuf, "Using likelihood-free inference to compare evolutionary dynamics of the protein networks of H. pylori and P. falciparum," PLoS Comput Biol, vol. 3, no. 11, pp. 2266-2276, 2007.

[15] M. Beaumont, W. Zhang, and D. Balding, "Approximate Bayesian Computation in Population Genetics," Genetics, vol. 162, no. 4, pp. 2025-2035, 2002.

[16] G. Peters, M. Wüthrich, and P. Shevchenko, "Chain Ladder Method: Bayesian Bootstrap versus Classical Bootstrap," Accepted for publication in Insurance: Mathematics and Economics.

[17] G. Peters, Y. Fan, and S. Sisson, "On sequential Monte Carlo, partial rejection control and approximate Bayesian computation," arXiv:0808.3466v2, 2008.

[18] S. Sisson, G. Peters, Y. Fan, and M. Briers, "Likelihood-free samplers "Preprint - UNSW statistics.

[19] S. Sisson and Y. Fan, "Likelihood-free Markov chain Monte Carlo," Arxiv preprint arXiv:1001.2058, 2010.

[20] P. Bortot, S. Coles, and S. Sisson, "Inference for Stereological Extremes," Journal-American Statistical Association, vol. 102, no. 477, pp. 84-92, 2007.

[21] O. Ratmann, C. Andrieu, C. Wiuf, and S. Richardson, "Model criticism based on likelihood-free inference, with an application to protein network evolution," Proceedings of the National Academy of Sciences of the United States of America, 2009.

[22] T. Toni, D. Welch, N. Strelkowa, A. Ipsen, and M. Stumpf, "Approximate Bayesian computation scheme for parameter inference and model selection in dynamical systems," Journal of The Royal Society Interface, vol. 6, no. 31, p. 187, 2009.

[23] G. Hamilton, M. Currat, N. Ray, G. Heckel, M. Beaumont, and L. Excoffier, "Bayesian estimation of recent migration rates after a spatial expansion," Genetics, vol. 170, no. 1, pp. 409-417, 2005.

[24] M. Tanaka, A. Francis, F. Luciani, and S. Sisson, "Using approximate Bayesian computation to estimate tuberculosis transmission parameters from genotype data," Genetics, vol. 173, no. 3, p. 1511, 2006.

[25] G. Peters and S. Sisson, "Bayesian inference, Monte Carlo sampling and operational risk," Journal of Operational Risk, vol. 1, no. 3, pp. 27-50, 2006.

[26] P. Marjoram, J. Molitor, V. Plagnol, and S. Tavare, "Markov chain Monte Carlo without likelihoods," Proceedings of the National Academy of Sciences, vol. 100, no. 26, pp. 15 324-15 328, 2003.

[27] R. R. W. and P. A. N., "A theoretical framework for approximate Bayesian computation." Proceedings of the International Workshop for Statistical Modelling, pp. 393-396, 2005.

[28] M. Blum, "Approximate Bayesian Computation: a non-parametric perspective," Arxiv preprint arXiv:0904.0635, 2009.

[29] P. Del Moral, A. Doucet, and A. Jasra, "An adaptive sequential Monte Carlo method for approximate Bayesian computation," preprint, 2009.

[30] V. Plagnol and S. Tavaré, Approximate Bayesian computation and MCMC. Springer Verlag, 2004.

[31] S. Chib and E. Greenberg, "Understanding the Metropolis-Hastings algorithm," The American Statistician, vol. 49, no. 4, pp. 327-335, 1995.

[32] W. Gilks and D. Spiegelhalter, Markov chain Monte Carlo in practice. Chapman \& Hall/CRC, 1996. 